\title{
NON-LINEAR CONTROL OF FOUR-WHEEL STEERING CARS WITH ACTUATOR CONSTRAINTS
}

\author{
Miguel A. Vilaplana ${ }^{\text {}}$, Oliver Mason ${ }^{1}$, Douglas J. Leith ${ }^{1}$, William E. Leithead ${ }^{1}$, Jens Kalkkuhl ${ }^{2}$ \\ 'Hamilton Institute, NUI Maynooth, Co. Kildare, Ireland \\ ${ }^{2}$ DaimlerChrysler AG Research \& Technology, Stuttgart, Germany
}

\begin{abstract}
This paper presents a new non-linear steering controller for cars equipped with 4-wheel steer-by-wire that tracks reference sideslip and yaw rate signals describing the desired lateral dynamics. The proposed controller automatically rejects any disturbances in sideslip and yaw-rate, and incorporates an anti-windup scheme to reduce the effects of the saturation of the rear steering actuators. The control design is based on the Individual Channel Analysis and Design (ICAD) methodology. An analysis of the robust stability of the control system is presented. Results from a detailed non-linear simulation model are given to illustrate the controller's performance. Copyright (c) 2004 IFAC
\end{abstract}

Keywords: Vehicle dynamics, Automotive control, Actuator saturation, Integrator windup, Stability analysis.

\section{INTRODUCTION}

This paper presents a new non-linear steering controller for 4-wheel steering cars. The proposed controller commands the front and rear steering angles with the objective of tracking reference sideslip and yaw rate signals obtained from the driver's inputs to steering wheel and pedals. These reference signals describe the desired lateral dynamics for the controlled car. In addition, the steering controller automatically rejects any disturbances in sideslip and yaw rate caused, for example, by lateral gusts of wind or $\mu$-split braking manoeuvres. It is assumed that the controlled output variables, i.e. sideslip angle and yaw rate, are measured (in practice, the latter might typically be estimated using, for example, a Kalman filter).

There is already a substantial body of research on the control of 4-wheel steering cars and a variety of control structures have been proposed (see, e.g. Ackermann, 1994 and Koumboulis and Skarpetis, 2002). The controller proposed here aims to achieve satisfactory performance and robustness taking into account the possible saturation of the rear steering actuators during operation. The issue of rear actuator saturation has not been fully investigated in the literature.

The structure of the steering controller presented in this paper is based on a simplified linear model of the lateral dynamics of 4-wheel steering cars at constant speed. The main elements of the controller structure are a linear input transformation and a speeddependent feedback. These elements result in the partial decoupling of the sideslip and yaw rate responses with respect to the transformed inputs and yield speed-invariant yaw rate dynamics, thereby acting as an implicit gain scheduling on the vehicle speed. A more accurate model of the dynamics of 4wheel steering cars is then considered. When applied to this new model, the proposed controller structure results in approximate partial decoupling and nearly speed-invariant yaw rate dynamics. The originally 2 by- 2 multivariable control design problem can then be broken down into two SISO control design problems according to the ICAD paradigm (O'Reilly and Leithead, 1991). Assuming certain bandwidth restrictions, controllers for the resulting sideslip and yaw rate channels can be easily designed within the 
proposed structure. The resulting controllers satisfy disturbance rejection requirements. A feedforward element is introduced to improve the response to reference inputs. Finally, an anti-windup scheme is incorporated into the controller to mitigate the effects of the saturation of the rear steering actuators. The resulting nonlinear steering controller is valid for varying vehicle speed and shows an excellent degree of robustness to plant parameter uncertainty.

The remainder of this paper is structured as follows. First the simplified model of the lateral dynamics of a 4-wheel steering car used to define the proposed steering controller structure is presented. Next, the steering controller structure is described based on this model. The proposed structure is then applied to a more accurate model of the car lateral dynamics and a feedback controller valid for varying vehicle speed designed following the ICAD approach is presented. The resulting controller is augmented with a feedforward element, which improves the responses to reference inputs, and with an antiwindup scheme, which allows the controller to cope with rear actuator saturation. The robust stability of the resulting non-linear feedback controller is then analysed. Finally, simulation results obtained with a detailed non-linear model of a 4-wheel steering Mercedes S-Class are given to illustrate the performance of the controller.

\section{SIMPLIFIED LINEAR MODEL OF THE LATERAL DYNAMICS OF 4-WHEEL STEERING CARS AT CONSTANT SPEED}

Throughout this paper, it is assumed that the essential features of the lateral dynamics of the car can be described using the single-track model [3]. According to the single-track model, the two wheels at each axle are lumped into a single imaginary wheel located at the centre of the respective axle. The resulting front and rear wheels are interconnected by a one-dimensional rigid element with the car's mass distribution. Only the lateral motion of the car is considered when using the single-track model. It is assumed that the car's speed is constant and that the sideslip and steering angles remain small. Figure 1 depicts the single-track model indicating the main elements necessary for the analysis of the lateral dynamics.

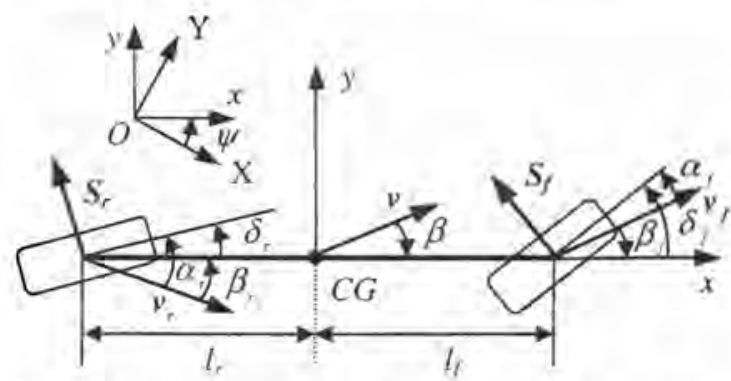

Fig. 1. Single-track model of a 4-wheel steering car.

In Figure 1, CGxyz are a set of reference axes fixed to the vehicle with origin at the centre of mass $C G, v$ is the velocity of the car with respect to the inertial reference $O X Y Z, v_{f}$ and $v_{r}$ are the velocities at the front and rear axles, respectively, with respect to $O X Y Z, \psi$ is the yaw angle and $\beta$ is the sideslip angle. It is assumed that the two wheels at each car's axle are steered to the same angle ( $\delta$ and $\delta_{r}$ in Figure 1). The only external forces acting on the single-track model are $S_{f}$ and $S_{r}$ depicted in Figure I, which represent the cornering forces generated by the interaction between the tyres and the road surface. It is assumed that $S_{f}$ and $S_{r}$ are linear on the tyre sideslip angles ( $\alpha_{f}$ and $\alpha_{1}$ in Figure 1):

$$
S_{f}=K_{f} \alpha_{f}, S_{r}=K_{r} \alpha_{r}
$$

The constant $K_{f}$ is the result of modifying the combined stiffness of the two front tyres to take into account the caster effect of the steering system at the front axle. The constant $K_{r}$ is simply the combined cornering stiffness of the rear tyre as it is assumed that no caster effect is generated in the steering of the rear wheels.

The following LTI state-space equations are obtained by linearising the equations of motion applied to the single-track model about the equilibrium point given by zero sideslip, zero yaw rate and zero steering angles:

$$
\begin{gathered}
\dot{x}=A x+B u \\
y=C x+D u \\
{\left[\begin{array}{c}
\delta_{f} \\
\delta_{r}
\end{array}\right], y=x=\left[\begin{array}{c}
\beta \\
\dot{\psi}
\end{array}\right],} \\
A=\left[\begin{array}{cc}
-\frac{K_{f}+K_{r}}{m v_{x}} & \frac{K_{f} l_{f}-K_{r} l_{r}}{m v_{r}^{2}}+1 \\
\frac{K_{f} l_{f}-K_{r} l_{r}}{I_{z z}} & -\frac{K_{f} l_{f}^{2}+K_{r} l_{r}^{2}}{I_{z} v_{x}}
\end{array}\right] . \\
B=\left[\begin{array}{cc}
-\frac{K_{f}}{m v_{x}} & -\frac{K_{r}}{m v_{x}} \\
\frac{K_{f} l_{f}}{I_{z z}} & -\frac{K_{r} l_{r}}{I_{z}}
\end{array}\right], C=\left[\begin{array}{ll}
1 & 0 \\
0 & 1
\end{array}\right], D=\left[\begin{array}{ll}
0 & 0 \\
0 & 0
\end{array}\right]
\end{gathered}
$$

In these equations, $m$ is the mass of the vehicle, $I_{z z}$ is its moment of inertia with respect to the $C G-z$ axis and $v_{r}$ is the projection of the vehicle speed along the $C G-x$ axis (hereafier referred to simply as the vehicle speed). The transfer function corresponding to the state-representation above is:

$$
G(s)=C(s I-A)^{-1} B+D=\left[\begin{array}{ll}
g_{11}(s) & g_{12}(s) \\
g_{21}(s) & g_{22}(s)
\end{array}\right]
$$

\section{CONTROLLER STRUCTURE}

The control design problem is stated in terms of a new plant model that results from modifying $G(s)$ using input transformation and speed-dependent feedback based on the dynamical equations (2). The resulting plant to be controlled, $\widetilde{G}(s)$, is uppertriangular and yields speed-invariant yaw rate 
dynamics. Expressing the problem in terms of $\tilde{G}(s)$ instead of $G(s)$ and assuming diagonal control greatly simplifies the design task. The proposed controller structure is explained below.

\subsection{Input transformation.}

Suppose the inputs to the plant $G(s)$ are the result of the following linear transformation:

$$
\left[\begin{array}{l}
\delta_{f} \\
\delta_{r}
\end{array}\right]=E\left[\begin{array}{l}
\Delta_{1} \\
\Delta_{2}
\end{array}\right]
$$

with $E$ a matrix with constant entries. Considering (4), the resulting state dynamical equation for the single-track model with respect to the new inputs is:

$$
\dot{x}=A x+B E\left[\begin{array}{l}
\Delta_{1} \\
\Delta_{2}
\end{array}\right]=A x+B_{1}\left[\begin{array}{l}
\Delta_{1} \\
\Delta_{2}
\end{array}\right]
$$

Choosing

$$
E=-\frac{1}{\frac{K_{r}}{K_{f}}\left(1+\frac{l_{r}}{l_{f}}\right)}\left[\begin{array}{cc}
\frac{K_{r} l_{r}}{K_{f} l_{f}} & -\frac{K_{r}}{K_{f}} \\
-1 & -1
\end{array}\right]
$$

results in $B_{1}$ diagonal:

$$
B_{1}=\left[\begin{array}{cc}
-\frac{K_{f}}{m v_{x}} & 0 \\
0 & \frac{K_{f} l_{f}}{I_{z z}}
\end{array}\right]
$$

The chosen matrix $E$ correspond to the inputs:

$$
\Delta_{1}=\delta_{f}+\frac{K_{r}}{K_{f}} \delta_{r}, \Delta_{2}=\delta_{f}-\frac{K_{r} l_{r}}{K_{f} l_{f}} \delta_{r}
$$

A physical interpretation of these new inputs is in terms of a mode given by $\Delta_{1}$, where the front and rear wheels steer in the same direction and a mode given by $\Delta_{2}$, where the front and rear wheels steer in opposite directions.

The resulting dynamical equation of the yaw rate with respect to the new inputs is:

$$
\frac{l_{z}}{K_{f} l_{f}} \ddot{\psi}+\frac{K_{f} l_{f}^{2}+K_{r} l_{r}^{2}}{K_{f} l_{f} v_{x}} \dot{\psi}=\Delta_{2}+\left(1-\frac{K_{r} l_{r}}{K_{f} l_{f}}\right) \beta
$$

Taking Laplace transforms in (9) results in:

$$
\dot{\psi}(s)=\frac{K_{1}}{s+p\left(v_{x}\right)} \Delta_{2}(s)+\frac{K_{1} K_{2}}{s+p\left(v_{s}\right)} \beta(s)
$$

with

$$
K_{1}=\frac{K_{f} l_{f}}{l_{z}}, K_{2}=1-\frac{K_{r} l_{r}}{K_{f} l_{f}}, p\left(v_{x}\right)=\frac{K_{f} l_{f}^{2}+K_{r} l_{r}^{2}}{I_{z} v_{x}}(11)
$$

\subsection{Speed-dependent feedback.}

The following feedback element is introduced:

$$
\left[\begin{array}{c}
\Delta_{1} \\
\Delta_{2}
\end{array}\right]=\left[\begin{array}{c}
\tilde{\Delta}_{1} \\
\tilde{\Delta}_{2}
\end{array}\right]-\left[\begin{array}{cc}
0 & 0 \\
K_{2} & K_{v}\left(v_{x}\right)
\end{array}\right]\left[\begin{array}{l}
\beta \\
\dot{\psi}
\end{array}\right]
$$

where $K_{v}\left(v_{x}\right)$ is defined as:

$$
K_{v}\left(v_{x}\right)=K_{0}-\frac{p\left(v_{x}\right)}{K_{1}}
$$

with $K_{0}$ an arbitrary constant. The resulting state equations are:

$$
\dot{x}=\tilde{A} x+B_{1}\left[\begin{array}{l}
\tilde{\Delta}_{1} \\
\tilde{\Delta}_{2}
\end{array}\right]
$$

where

$$
\tilde{A}=\left[\begin{array}{cc}
-\frac{K_{f}+K_{r}}{m v_{x}} & \frac{K_{f} l_{f}-K_{r} l_{r}}{m v_{x}^{2}}+1 \\
0 & -\frac{K_{0} K_{f} l_{f}}{I_{z z}}
\end{array}\right]
$$

The corresponding transfer function with respect to the new inputs, $\left[\begin{array}{c}\tilde{\Delta}_{1}(s) \\ \tilde{\Delta}_{2}(s)\end{array}\right]$, is upper-triangular:

$$
\tilde{G}(s)=C(s I-\tilde{A})^{-1} B_{1}+D=\left[\begin{array}{cc}
\tilde{g}_{11}(s) & \tilde{g}_{12}(s) \\
0 & \tilde{g}_{22}(s)
\end{array}\right]
$$

The dynamical equation of the yaw rate with respect to the inputs is:

$$
\ddot{\psi}=-K_{0} K_{1} \dot{\psi}+K_{1} \tilde{\Delta}_{2}
$$

If $K_{0}$ is chosen to be:

$$
K_{0}=\frac{K_{f} l_{f}^{2}+K_{r} l_{r}^{2}}{K_{f} l_{f} v_{x 0}}
$$

where $v_{x 0}$ is a constant with the value of an arbitrary chosen vehicle speed, then taking Laplace transforms in (17) results in:

$$
\dot{\psi}(s)=\frac{K_{1}}{s+p\left(v_{x 0}\right)} \tilde{\Delta}_{2}(s)
$$

The introduction of the feedback element described above results in the yaw rate dynamics depending only on one of the two inputs to be controlled, $\tilde{\Delta}_{2}(s)$. Besides, the yaw rate response to $\tilde{\Delta}_{2}(s)$ is speed-invariant and characterised by the fixed pole $p\left(v_{x 0}\right)$ in (19), which can in principle be arbitrarily selected by assigning a value to $v_{x 0}$ in $K_{0}$.

3.3 Control design problem with diagonal controller.

It is assumed, for simplicity, that the plant $\widetilde{G}(s)$ is to be controlled by a diagonal controller. Thus, with the proposed controller structure in place the 
resulting multivariable control design process is reduced to the design of the linear controllers $\tilde{k}_{1}(s)$ and $\vec{k}_{2}(s)$ (see Figure 2).

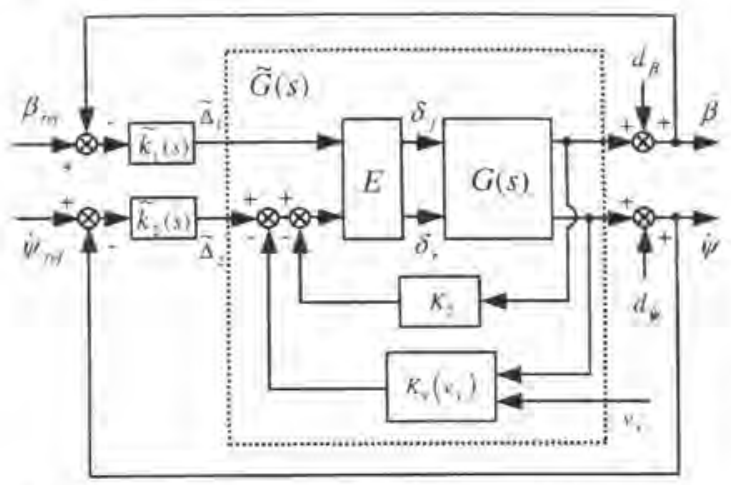

Fig. 2: Multivariable control design problem.

\section{CONTROL DESIGN}

The controller structure described in the previous section has been applied to the design of the controllers $\tilde{k}_{1}(s)$ and $\tilde{k}_{2}(s)$ for a more accurate linear model of the car lateral dynamics at constant spced. This model, which still relies on the singletrack approximation, takes into account some additional features of the dynamics of a 4-wheel steering car, such as the front and rear actuator dynamics (modelled as second or systems), the dynamics of the tyre forces and the interaction of the tyre forces with the caster effect.

The controllers $\tilde{k}_{1}(s)$ and $\tilde{k}_{2}(s)$ in Figure 2 are designed based on the virtual plant $\tilde{G}(s)$ that results from replacing $G(s)$ with the transfer function matrix of the new model introduced above. Considering the nominal physical parameters of a Mercedes S-Class, the resulting $\tilde{G}(s)$ can still be considered upper-triangular with speed-independent yaw rate dynamics up to frequencies around $10 \mathrm{rad} / \mathrm{s}$. By adopting the ICAD approach and imposing a bandwidth separation between the resulting sideslip and yaw rate channels, simple controllers $\tilde{k}_{1}(s)$ and $\tilde{k}_{2}(s)$ can be designed individually using linear SISO techniques. Linear controllers of the form

$$
\begin{aligned}
& \tilde{k}_{1}(s)=-\frac{1}{s T_{11}} \text { (I controller) } \\
& \tilde{k}_{2}(s)=K_{P 2}+\frac{1}{s T_{12}} \text { (PI controller) }
\end{aligned}
$$

can easily be tuned to obtain satisfactory robustness margins and disturbance rejection performance with a low bandwidth sideslip channel (approx $3 \mathrm{rad} / \mathrm{s}$ ) and a high bandwidth yaw rate channel (approx 10 $\mathrm{rad} / \mathrm{s}$ ). Assuming slow-varying vehicle speed, the speed-dependent feedback term $K_{v}\left(v_{x}\right)$ acts as an implicit gain scheduling scheme that results in a non- linear controller comprised of linear controllers parameterised by the vehicle speed.

A linear feedforward element is incorporated into the sleering controller with the objective of speeding up and shaping the response to reference signals.

\section{ANTI-WINDUP SCHEME}

Since the rear wheels can only be steered to a very small angle due to space constraints and the steering controller performs integrating action, an antiwindup scheme has been devised to cope with the effects of the saturation of the rear actuators. The proposed scheme is inspired by conventional antiwindup methods and works as follows. The rear steering angle signal commanded by the controller is suburacted from the measured rear steering angle. The resulting signal is fed to the input of the controller $\tilde{k}_{1}$ through a gain $K_{A W}$. As it will be shown in the simulation results below, this scheme prevents the integrators in both $\tilde{k}_{1}$ and $\tilde{k}_{2}$ from winding up and allows the steering controller to retain control of the car. Figure 3 schematically shows the full steering controller, including feedforward and anti-windup, as it would be implemented in a real car.

\section{ROBUST STABILITY ANALYSIS}

It is possible to write the feedback part of the overall control system (based on the more accurate model of the lateral dynamics, and incorporating the antiwindup scheme described above) in the form depicted in Figure 4 . In the figure, $\delta_{r, r}$ is the rear steering angle demanded by the controller and $\delta_{r}$ is the actual rear steering angle, and $b, c$ are vectors. When written in this way, our system is an example of a Lur'e system, and stability results, such as the Circle Criterion (Narendra and Goldwyn, 1964; Willems, 1973), that have been developed for such systems can be used to analyse the system.

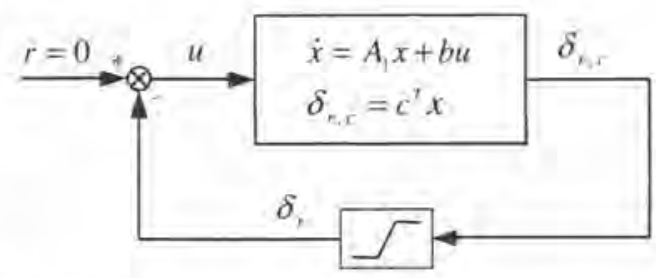

Fig 4. Closed loop system for stability analysis.

The closed loop state equation now takes the form

$$
\dot{x}=\left(A_{1}-k_{n I}\left(\delta_{r, c}\right) b c^{T}\right) x
$$

The non-linear function $k_{n l}\left(\delta_{r, c}\right)$ represents the saturation non-linearity and is given by: 


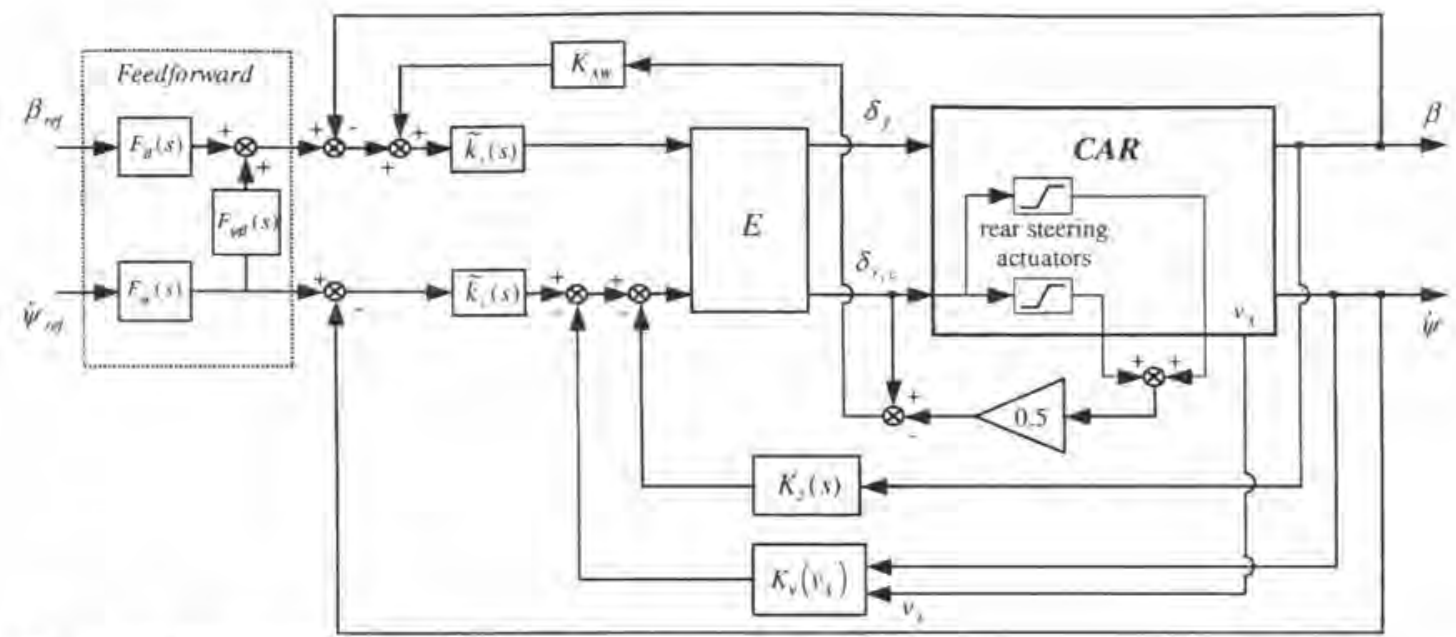

Fig 3. Full steering controller.

$$
k_{n l}\left(\delta_{r, c}\right)=\left\{\begin{array}{ccc}
1 & \text { if } & \left|\delta_{r . c}\right| \leq \delta_{\max } \\
\delta_{\max } & \text { if } & \left|\delta_{r, c}\right|>\delta_{\max }
\end{array}\right.
$$

Here $\delta_{\text {max }}$ is the absolute value of the maximum rear steering angle allowed by the actuators. Note that the function $k_{n l}\left(\delta_{r, c}\right)$ can be written as a function of the state and $0 \leq k_{n \prime}(x) \leq 1$ for all $x$. Now, if there is a positive definite matrix $P$ such that

$$
A_{1}^{T} P+P A_{1}<0,\left(A_{1}-b c^{T}\right)^{T} P+P\left(A_{1}-b c^{T}\right)<0
$$

then $V(x)=x^{T} P x$ will define a Lyapunov function for the system (21), thus assuring its asymptotic stability. This follows because all of the matrices $A_{4}-k(x) b c^{T}$ that arise in (21) are convex combinations of the two matrices $A_{1}, A_{1}-b c^{T}$. Thus if there is a solution $P$ to (23), this guarantees the asymptotic stability of (21).

The Circle Criterion provides a frequency domain condition that can be used to test for the existence of a solution to (23). It has recently been shown that it is also possible to test for the existence of such a solution using a simple time-domain condition (Shorten and Narendra, 2003; Shorten et al, 2004). Specifically, there is a positive definite $P$ satisfying (23) if and only if the matrix $A_{1}\left(A_{1}-b c^{T}\right)$ has no negative real eigenvalues. The stability and robustness of the non-linear system in Figure 4 with respect to parametric uncertainty are analysed using this fact. A major advantage of the time-domain condition is its simplicity, as it only requires the calculation of one set of eigenvalues as opposed to checking a frequency domain condition for infinitely many values of a variable.

Figure 5 below is generated as follows. First, the steering controller is tuned for the nominal values of the model parameters corresponding to a Mercedes S-Class. The real values of those parameters are uncerlain, each of them lying within an interval

around its nominal value. For a given fixed vehicle speed, we calculate the entries of $A_{1}, b$ and $c$ for a large number of values of the car model parameters randomly chosen from their respective uncertainty intervals. We then calculate the eigenvalues of $A_{1}\left(A_{1}-b c^{r}\right)$ for all the values of the parameters considered. We repeat the process outlined above for three different vehicle speeds $(20,35$ and $50 \mathrm{~m} / \mathrm{s})$. Figure 5 shows the two eigenvalues of $A_{1}\left(A_{1}-b c^{T}\right) \quad$ closest to the real axis obtained with the different random values of the car model parameters for the three speeds considered. Since those eigenvalues can be seen to remain well clear of the negative real axis, we can conclude that the system in Figure 4 is robustly asymptotically stable for the three speeds chosen.

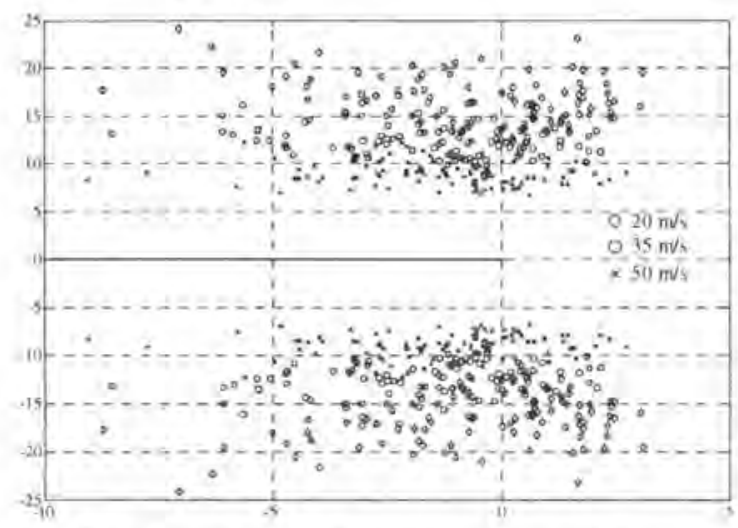

Fig. 5. Illustration of robust asymptotic stability.

\section{SIMULATION RESULTS}

The full steering controller has been discretised and its performance has been simulated using a detailed non-linear simulation model of a Mercedes S-Class. The model includes a communication delay between controller and actuators of $20 \mathrm{~ms}$. The following manoeuvre is considered. Suppose that the car travels on a road with a $u$-split surface so that the two wheels at the left hand side of the car are on dry asphalt $(\mu \approx 1)$ and the two on the right hand side are 
on ice $(\mu \approx 0.2)$. While turning at $50 \mathrm{~m} / \mathrm{s}$, the driver applies the brakes moderately for 1 second (between time $=8 \mathrm{~s}$ and time $=9 \mathrm{~s}$ ). The simulation results shown in Figure 6 below illustrate the response of the controlled car with and without anti-windup. The steering controller attempts to automatically reject the disturbances introduced by braking while tracking the reference sideslip and yaw rate. This results in the saturation of the rear actuators. Without anti-windup, the controller is not able to recover from the disturbances and spin out of control. On the other hand, the full steering controller (with anti-windup) is able to retain control of the car. These results show the ability of the proposed non-linear steering controller to perform under simultaneous braking and turning on a $\mu$-split surface, demonstrating the robustness of the control system for varying vehicle speed.

\section{CONCLUSIONS}

This paper has presented a steering controller whose structure is based on the basic physics of 4-wheel steering. The controller incorporates an anti-windup scheme to mitigate the effects of the possible saturation of the rear steering actuators. The robust stability of the resulting non-linear control system has been demonstrated using tools from non-linear systems analysis and its robustness has been illustrated through simulation. Future work will include a detailed robustness and integrity analysis together with validation experiments on a car equipped with 4-wheel steer-by-wire.

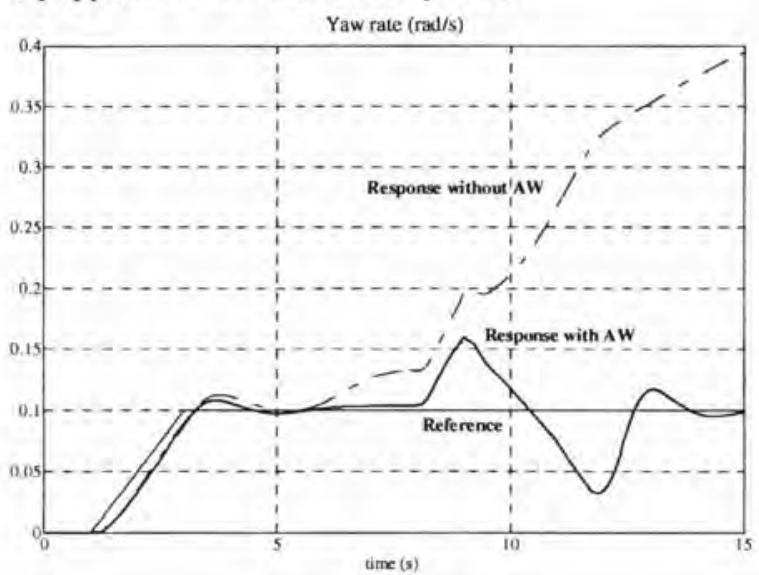

Vehicle speed ( $\mathrm{m} / \mathrm{s}$ )

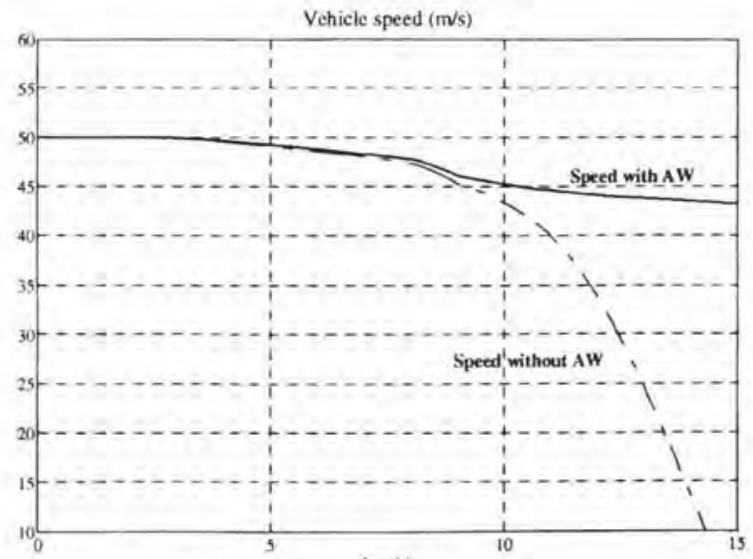

Fig. 6. Simulation results.

\section{REFERENCES}

Ackermann, J. (1994). Robust decoupling, ideal steering dynamics and yaw stabilization of 4WS cars. Automatica, 30(11) , 1761-1768.

Koumboulis, F. N. and M. G. Skarpetis (2002), Robust control of cars with front and rear wheel steering, IEE Proceedings - Control Theory Applications, 149(5), 394-404.

Narendra, K.S. and R. Goldwyn (1964). A geometrical criterion for the stability of certain non-linear, non-autonomous systems. IEEE Transactions on Circuit Theory, 11, 406-407.

O'Reilly, J. and W. E. Leithead (1991), Multivariable control by 'individual channel design', International Journal of Control, 54(1), 1-46.

Shorten, R.N. and K.S. Narendra (2003). On common quadratic Lyapunov functions for pairs of stable LTI systems whose system matrices are in companion form. IEEE Transactions on Automatic Control, 48(4), 618-621.

Shorten, R.N., O. Mason, F. O'Cairbre and P. Curran (2004). A unifying framework for the SISO circle criterion and other quadratic stability criteria. International Journal of Control, 77(1), $1-8$.

Willems, J. (1973). The circle criterion and quadratic Lyapunov functions for stability analysis. IEEE Transactions on Automatic Control, 18, 184.
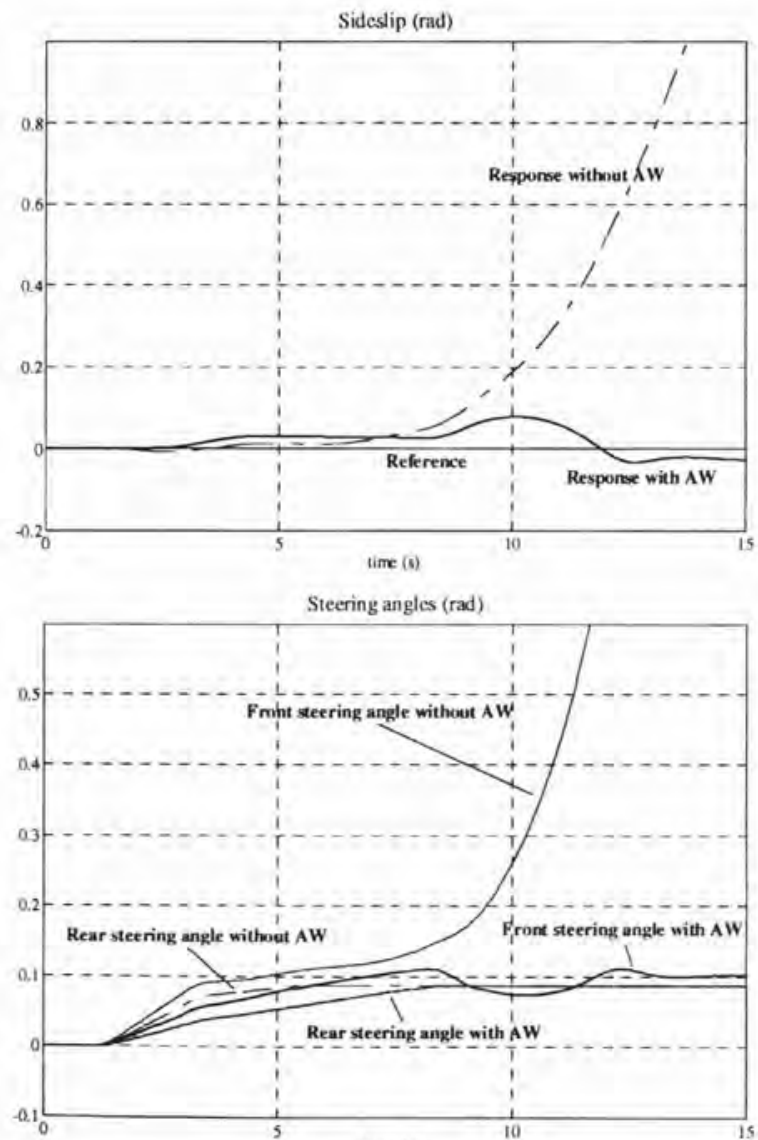

$\tan (3)$ 